\title{
Neuroanatomical basis for first- and second-order representations of bodily states
}

\author{
H. D. Critchley ${ }^{1,2}$, C. J. Mathias ${ }^{2,3}$ and R. J. Dolan ${ }^{1}$ \\ 1 Wellcome Department of Cognitive N eurology, Institute of Neurology, University College London, 12 Queen Square, London WC1N 3BG, UK \\ 2 Autonomic Unit, National H ospital for Neurology \& Neurosurgery and Institute of Neurology, University College London, Queen Square, London WC1N 3BG, UK \\ ${ }^{3}$ Neurovascular M edicine Unit, Saint M ary's H ospital, ICSM , Praed Street, London W2 INY, UK \\ Correspondence should be addressed to R.J.D. (r.dolan@fil.ion.ucl.ac.uk)
}

\begin{abstract}
Changes in bodily states, particularly those mediated by the autonomic nervous system, are crucial to ongoing emotional experience. A theoretical model proposes a first-order autoregulatory representation of bodily state at the level of dorsal pons, and a second-order experience-dependent re-mapping of changes in bodily state within structures such as cingulate and medial parietal cortices. We tested these anatomical predictions using positron emission tomography and a human neurological model (pure autonomic failure), in which peripheral autonomic denervation prevents the emergence of autonomic responses. Compared to controls, we observed task-independent differences in activity of dorsal pons and context-induced differences in cingulate and medial parietal activity in PAF patients. An absence of afferent feedback concerning autonomically generated bodily states was associated with subtle impairments of emotional responses in PAF patients. Our findings provide empirical support for a theory proposing a hierarchical representation of bodily states.
\end{abstract}

Emotional, cognitive and physical behaviors mediated by the central nervous system are integrated with peripheral changes in bodily states. Moreover, physiological changes in bodily state directly influence emotional expression $n^{1,2}$ and cognitive functions such as decision-making ${ }^{3}$ and memory 4 . At a broader level, emotions may be subsumed within homeostatic mechanisms that underlie survival of the organism ${ }^{2,5}$. In a theoretical model ${ }^{5}$ based on neurological observations, prime emphasis is given to the cerebral representation of bodily states as a substrate for emotions and conscious awareness. Fundamental components of the model are first-order autoregulatory mappings and second-order re-mappings of bodily states consequent upon behavioral experience. The dorsal pons is proposed to support first-order mapping of bodily state, because of its involvement in homeostatic regulation mediated via afferent information from the body. The model also proposes that somatosensory areas, the somatosensory cortices and insula, contribute to this primary representation of bodily states. In contrast, cingulate and medial parietal cortices are critical components in second-order mappings of experience-dependent changes in body states.

Here we tested specific predictions arising from these theoretical considerations. We compared the state-dependent regional brain activity in healthy human subjects with activity in subjects with pure autonomic failure $(\mathrm{PAF})^{6}$. PAF is an idiopathic disorder acquired in middle age that only affects the peripheral autonomic nervous system. Subjects with PAF cannot modulate their bodily state via the autonomic nervous system because of peripheral autonomic denervation, but have no other neurolog- ical deficit (sensory or motor). Consequently, there is no integrated central feedback of information concerning autonomic changes that are normal accompaniments of ongoing behavior ${ }^{6}$. Peripheral autonomic denervation results in a failure of neurogenic control of circulation, the cardinal feature of which is orthostatic (postural) hypotension due to an inability to activate sympathetic pathways to engender vasoconstriction during gravitational challenges ${ }^{6-8}$. Additionally, subjects with PAF do not generate heart rate and blood pressure increases during effortful exercise or mental arithmetic (stressor tasks) ${ }^{6}$ and do not increase circulating catecholamines during physical or emotional challenge ${ }^{6}$. These subjects have absent sympathetic skin conductance responses to emotive and orienting stimuli ${ }^{9}$, and have diminished pupilliary reflexes ${ }^{10}$. Through histological evidence, PAF has been associated with Lewy bodies in peripheral autonomic ganglia ${ }^{11}$, but its cause is unknown ${ }^{6}$. PAF is distinguished from central neurodegenerative causes of autonomic failure (such as multiple system atrophy or Parkinson's disease with autonomic failure) by normal life expectancy ${ }^{6,7}$, and by the absence of clinical and hormonal ${ }^{12}$ indicators of central neurological degeneration. Similarly, gross and pervasive disturbances in attention or emotional functioning have not been documented during clinical observations, although subtle abnormalities in subjective emotional experience may exist. Consequently, PAF provides a unique lesion-deficit model to examine the involvement of specific brain areas in the first and second-order representation of bodily states.

We used positron emission tomography (PET) to compare neural activity in nine subjects with PAF with activity in eight 
a Change
in MAP $(\mathrm{mmHg})$

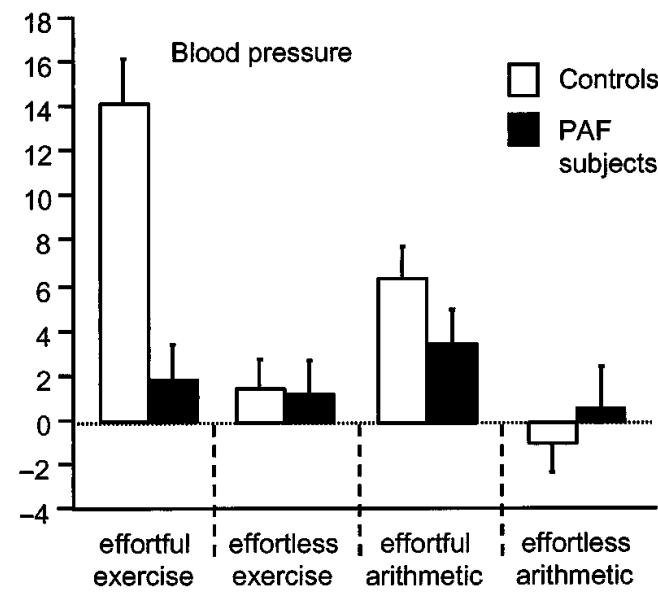

b

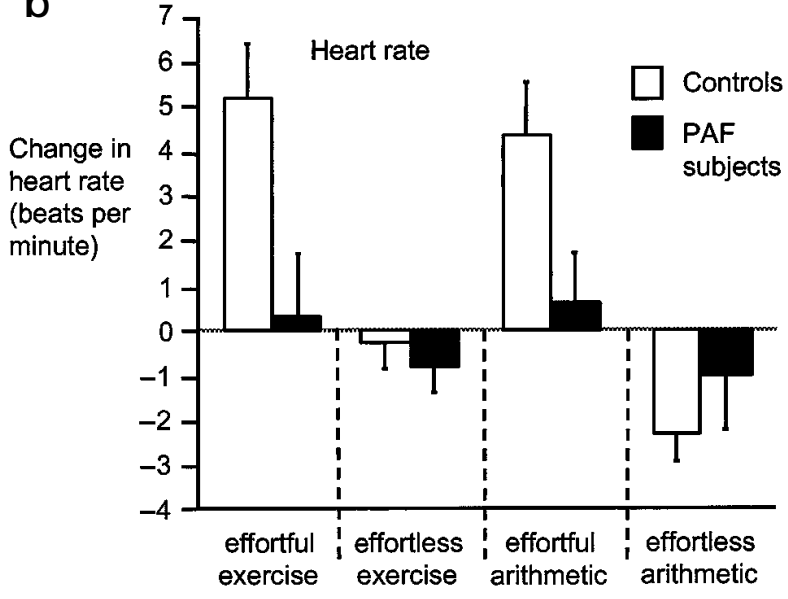

Fig 1. Physiological changes during task performance. (a) Group data (mean and standard error) showing changes in mean arterial blood pressure (MAP) during performance of effortful stressor tasks and corresponding low-grade tasks in controls (white bars) and subjects with PAF (black bars). Blood pressure differed between stressor and low-grade tasks in controls $\left(p<0.005\right.$, for exercise tasks, $t_{41}=4.94$; for arithmetic tasks $\left.t_{40}=3.19\right)$, but not PAF subjects ( $p>0.05$, for exercise tasks, $t_{40}=0.24$; for arithmetic tasks $t_{40}=1.13$ ). There was a significant task-by-diagnosis interaction $\left(F_{3}=6.8, p<0.001\right)$. (b) C hanges in heart rate during performance of stressor tasks and corresponding low-grade tasks in controls (white bars) and PAF subjects (black bars). Heart rate differed between stressor and low-grade tasks in controls ( $p<0.005$, for exercise tasks, $t_{41}=3.45$; for arithmetic tasks $\left.t_{40}=4.48\right)$, but not PAF subjects $\left(p>0.05\right.$, for exercise tasks, $t_{40}=0.57$; for arithmetic tasks $\left.t_{40}=0.93\right)$. There was a significant task-bydiagnosis interaction, $\left.F_{3}=3.0, p<0.05\right)$.

matched controls (healthy normal subjects) during performance of stressor and control tasks. Two types of these tasks were done, isometric handgrip and covert mental arithmetic, to examine commonalities in functional neuroanatomy associated with autonomic responses that are independent of systems dedicated to motor execution ${ }^{13}$ and phonological processing ${ }^{14}$. Lowgrade control conditions were also done (light handgrip and counting) as a baseline for each class of stressor task ${ }^{15}$. Compared to controls, we observed task-independent differences in activity of dorsal pons and context-induced differences in cingulate and medial parietal activity in PAF. Our findings provide empirical support for a theory proposing a hierarchical representation of bodily states.

\section{RESULTS}

In control subjects, stressor tasks evoked increased sympathetic activity and decreased parasympathetic activity, raising blood pressure and heart rate ${ }^{6,15}$. Though PAF subjects reported similar effort ratings as controls during the stressor tasks, they did not experience increases from baseline heart rate or blood pressure (Fig. 1).

To identify neuroanatomical substrates supporting first-order representations of bodily state, wetested for task-independent differences in regional activity, by doing a conjunction analysis ${ }^{16,17}$ for group differences that were expressed across all four tasks. In this analysis, there was significantly greater activity in dorsal pons ( $p<0.05$, corrected) in PAF subjects as compared to controls a
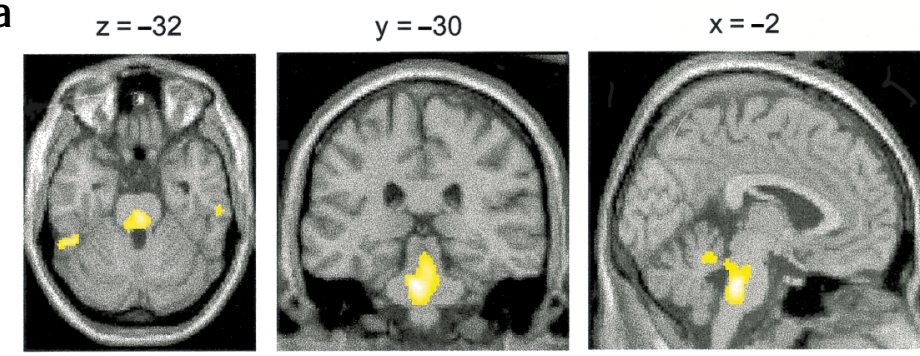

b

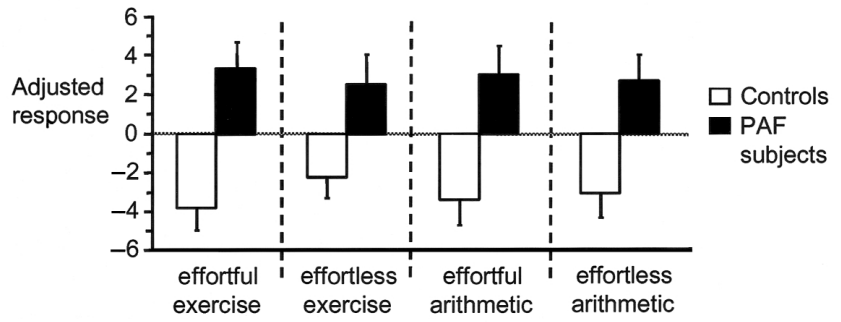

Fig. 2. Increased activity in pons in PAF subjects. (a) Significant between-group differences in regional cerebral activity determined by conjunction analysis across the four experimental tasks. Group data, reflecting significantly greater activity in dorsal pons (peak Talairach $x, y, z$ coordinates $37,-2,-30,-32$; $Z=6.83, p<0.05$ corrected) in PAF subjects than controls, is depicted on axial, coronal and parasaggital sections of a standard template image derived from one subject. A bove these sections are $z, y$ and $x$ coordinates. (b) Cerebral blood flow responses in dorsal pons $(-2,-30,-32)$ for controls and PAF subjects during the four experimental conditions, illustrating the task independence of between-group differences in pontine activity. Adjusted data is shown on the $y$-axis, representing signal changes relative to global blood flow in each scan, corrected for in the analysis using proportional scaling to an arbitrary mean of 50 . 
(Fig. 2). This regional difference was expressed across all tasks and was therefore not confounded by groupby-task interactions (Fig. 3). Critically, this pontine region encompassed pontine reticular, periaqueductal and parabrachial nuclei, which are proposed as specific sites for first-order autoregulatory functions. Additionally, significant task-independent differences in activity between PAF subjects and controls were found in somatosensory cortices, right insula, right parietal lobe, caudate nucleus and extrastriate visual cortices ( $p<0.05$, corrected; Table 1 ).

Our second analysis addressed the question of regional brain activity representing context-specific changes in bodily states. We examined for differential group activity during tasks that normally cause changes in bodily states, through testing for an interaction between diagnosis and task. For betweengroup analysis, we used conjunction analysis to determine differences in regional brain activity that reflected effort-induced changes in bodily state (stressor versus low-grade tasks) that were common to both exercise and mental arithmetic tasks. PAF subjects had significantly less activity than controls ( $p<0.05$, corrected) in left medial parietal/posterior cingulate region during stressor versus low-grade tasks (Fig. 3b). Conversely, PAF subjects had significantly greater activity than controls in right anterior cingulate during stressor versus low-grade tasks ( $p<0.05$, corrected; Fig. 3a). We did not observe significant differences in the activity of thalamus or superior colliculus between PAF subjects and controls in analysis of contextual differences in regional activity, despite predictions that these brain areas contribute to second-order representations of bodily states (Table $\mathbf{1}$ ).

Our study focuses on the neuroanatomical predictions of a theoretical mode ${ }^{5}$. However, this model al so suggests that perturbations in the representation of bodily states of arousal may
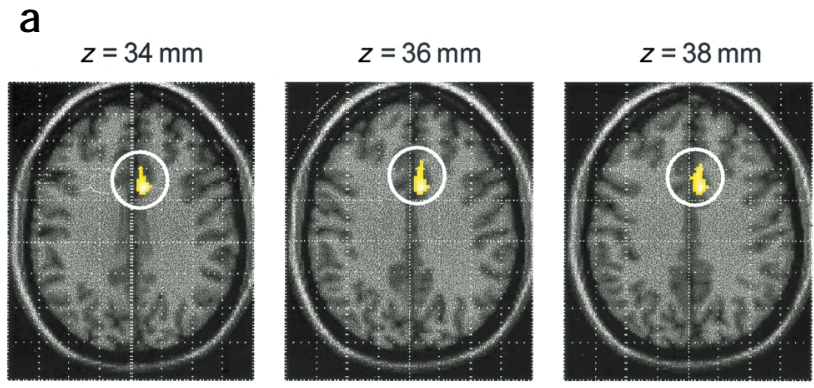

b $z=36 \mathrm{~mm}$

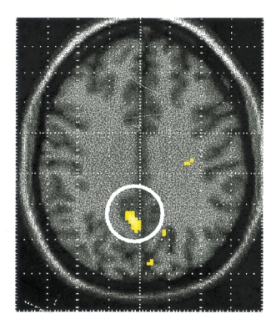

$z=38 \mathrm{~mm}$

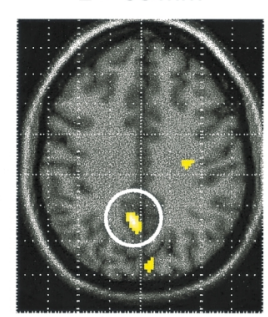

$z=40 \mathrm{~mm}$

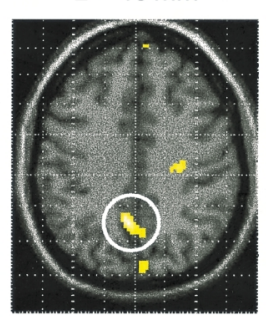

Table 1. Differences in regional brain activity between PAF subjects and controls: comparison with differences predicted by theoretical model.

\begin{tabular}{ll}
\hline $\begin{array}{l}\text { Regions supporting first-order representation of bodily state } \\
\text { Predicted }\end{array}$ & Observed* \\
Dorsal pons & D orsal pons $(-2,-30,-34)^{\ddagger}$ \\
Hypothalamus and basal forebrain & - \\
Somatosensory cortex & L somatosensory cortex $(-36,-12,42)^{\S}$ \\
& R somatosensory cortex $(-56,-20,22)^{\ddagger}$ \\
Insula & R insula $(32,14,0)^{\S}$ \\
- & R inferior parietal lobule $(44,-30,38)^{\S}$ \\
- & R superior parietal lobule $(38,-66,34)^{\S}$ \\
& Extrastriate visual cortices \\
& L middle temporal gyrus $(-52,-22,-4)^{\S}$ \\
& R fusiform gyrus $(56,-74,8)^{\S}$ \\
- & L lingual gyrus $(-12,-70,-4)^{\ddagger}$ \\
- & Caudate/putamen $(20,16,4)(-14,18,6)^{\ddagger}$ \\
& Cerebellum $(38,-42,-44)(-48,-44,-26)^{\ddagger}$
\end{tabular}

Regions supporting second-order representation of bodily state

\section{Predicted}

Cingulate/medial parietal lobe

Thalamus

Superior colliculus

${ }^{*} p<0.05$, corrected for whole brain, ${ }^{\dagger} p<0.05$ corrected for predicted regions.

₹ PAF > controls, ${ }^{\S}$ controls $>$ PAF.

\section{Observedt}

$\mathrm{R}$ anterior cingulate $(8,10,36)^{\S}$

L posterior cingulate $(-4,-56,38)^{\ddagger}$ influence emotional experience ${ }^{1,2,5}$. Thus, as a consequence of absent autonomic responses, PAF patients might be expected to show subtle emotional deficits. PAF patients were free of primary psychological disorder; thus, we tested for the possibility of subtle emotional change by rating PAF patients on scales of general disability ${ }^{18}$ and emotional function ${ }^{19}$. Although the PAF patients who underwent PET scanning were significantly disabled compared to healthy controls (mean \pm s.d., PAF patients versus controls, $6.8 \pm 3.5$ versus $\left.1.7 \pm 3.6, \mathrm{t}_{15}=2.8, \mathrm{p}<0.05\right)$, they did not experience expected symptoms of anxiety (PAF patients versus controls, $10.7 \pm 5.3$ versus $8.8 \pm 3.9, \mathrm{t}_{15}=0.89, \mathrm{p}=0.38$ ), even when more subjects than those in the imaging study were studied (PAF patients, $n=28$ versus controls, $n=15,11.3 \pm 5.9$ versus

Fig. 3. Between-group differences in activity: interaction with effort. (a) Significantly greater anterior cingulate activity (circled, peak $x, y$, $z$ coordinates, $8,10,36, z=4.48, p<0.05$, corrected for volume of regions predicted by the theoretical model to support second-order representations of bodily states, that is, cingulate, thalamus, superior colliculi in PAF subjects compared to controls, mapped onto three consecutive axial sections of a standard template image derived from one subject. Vertical $(z)$ coordinates are shown above each slice image. (b) Significantly greater posterior cingulate/medial parietal lobe activity (circled, peak $x, y, z$ coordinates, $-4,-56,38, z=4.51, p<0.05$, corrected for predicted regions) in controls relative to PAF subjects, mapped onto three consecutive axial sections of a standard template image derived from one subject. 
$8.7 \pm 3.3, t_{15}=1.6, p=0.12$ ). At clinical interview, PAF patients reported differences in their emotional reactions to stimuli and situations. On statements designed to probe subjective emotional experience, there was a trend for PAF patients to ratethemselves as feeling less emotional than controls, which was significant when the larger groups were compared (PAF patients versus controls, $3.3 \pm 0.6$ versus $\left.8.6 \pm 1.0, t_{41}=2.1, p<0.05\right)$. However, these differences in subjective affective experience could be attributable to physical disability, rather than autonomic failure per se. We therefore compared PAF patients to another group of neurological patients (people with idiopathic Parkinson's disease, IPD) who, unlike PAF patients, did not have clinically significant dysautonomia, but who were equally disabled (that is, the two groups were matched for disability). PAF patients were significantly less anxious than IPD patients (PAF versus IPD, $10.7 \pm 5.3$ versus $16.1 \pm 6.3, \mathrm{t}_{16}=2.2, \mathrm{p}<0.05$ ). M oreover, in larger samples of both PAF $(n=28)$ and IPD patients $(n=13)$, PAF patients had lower ratings of anxiety $(F=4.3, p<0.05)$ and rated morestrongly statements such as "I can no longer feel sad," and, "I have lost my ability to feel emotional," (respectively, $F=5.8, p<0.01$ and $F=10.8$, $p<0.01$ ) when disability scores were treated as confounding covariates. These latter statements explicitly reflected subjective emotional responses to situations.

\section{Discussion}

Our neuroimaging findings support the theory of a proposed hierarchical organization, and specified neural substrates involved in representing current and dynamically changing bodily states ${ }^{5}$. The wider relevance of this theoretical model lies in its suggestion that the most fundamental level of awareness ('proto-self') is supported by a first-order representation of bodily statein dorsal pontine nuclei controlling both wakefulness and homeostasis, and in regions such as insula and somatosensory cortex, which providea primary cortical representation of bodily states ${ }^{5,20}$. We observed differential activity in dorsal pons, right insula and somatosensory cortex associated with a pathological condition that leads (because of the peripheral autonomic denervation in PAF) to an uncoupling of central efferent control and afferent feedback of autonomic responses. In the pons, task-independent differential activity was present in a dorsal region that encompassed nuclei involved in control and representation of autonomic responses ${ }^{21}$, in particular, the parabrachial nucleus $s^{22,23}$ and periaqueductal gray ${ }^{24}$. Group differences in dorsal pons activity, commonly expressed across task, are consistent with a proposal that the pons has homeostatic involvement in autonomic regulation, and does not provide context-specific representation of peripheral arousal. Task-independent differences were also observed in somatosensory cortex and insula, regions proposed to support cortical mapping of bodily states ${ }^{5}$. In particular, there is evidence that theinsula is implicated in the representation of afferent visceral and autonomic information 15,25,26 and, in the right hemisphere, somatosensory cortex and insula are implicated in emotional representations ${ }^{27}$. M oreover, our findings in PAF subjects are consistent with the proposal that somatosensory regions contribute to a first-order, context-independent mapping of afferent visceral and autonomic information about bodily states ${ }^{5}$.

Task-independent regional differences between PAF and control subjects were evident in areas not predicted by the theoretical model. These regions included the caudate nucleus and right parietal, temporal, extrastriate visual and cerebellar cortices. Autonomic arousal modulates activity in right parietal lobe and extrastriate visual cortices ${ }^{28}$. The task-independent activity differences in these areas may reflect a functional reorganization in
PAF subjects resulting from lack of feedback modulatory influences from bodily states of autonomic arousal. We did not demonstrate task-independent differences in activity in basal forebrain and hypothalamus, which are also suggested to contribute to the first-order representations of bodily states. This may reflect the notion that the autoregulatory function of these regions is, with respect to hormonal regulation of bodily state, largely intact in PAF subjects.

The interaction between diagnosis and task (stressor versus low-grade tasks) enabled examination of the functional neuroanatomy underlying second-order representations that reflect experience-induced changes in bodily states. This second-order account provides a dynamic re-mapping of the physical state of theorganism in response to current behavioral and environmental contexts, drawing on representations of salient stimuli and events ${ }^{5}$. First-order representation may be influenced by the second-order account, as may sensory brain regions contributing information about objects and events relevant to it. The cingulate, using sensory, motor and attentional inputs, may fulfill this role through integration of information concerning bodily states 29,30 . The posterior cingulateand adjacent medial parietal lobe were significantly less active in PAF subjects than in controls for stressor versus control tasks. Both posterior cingulate and medial parietal cortex are putative components supporting the second-order account of organism/object relations ${ }^{5}$. Although the theoretical model does not explorethe different contributions of anterior and posterior cingulate regions to second-order mapping of bodily state, a functional reciprocity has previously been suggested between the 'evaluative' posterior cingulate and the 'executive' anterior cingulate ${ }^{30}$. The evaluative function of the posterior cingulate in monitoring sensory events (including in our experiment, peripheral autonomic arousal) is likely to be important for emotion and awareness. Our findings suggest that posterior cingulate/medial parietal lobe may provide the neural substrate for a mapping of the contextual relationship of bodily states.

In contrast to decreased posterior cingulate/medial parietal activity, increased anterior cingulate activity was manifest during effort (in PAF) when peripheral autonomic responses were not integrated with ongoing behavior. In other words, the absence of a mapping of afferent information about autonomic changes in bodily state is expressed in a relative increase in anterior cingulate activity. This right anterior cingulate area encompassed a region we previously identified as covarying with increases in measures of cardiovascular arousal in young healthy subjects ${ }^{15}$. Our observations in this pathological group are consistent with right anterior cingulate expressing an 'executive role' for the integration of autonomic responses with behavioral effort, reflecting dynamic reciprocity with the posterior cingulate/medial parietal region. We suggest that the anterior cingulate is abnormally active in PAF when representations in posterior cingulate of afferent information about changes in bodily states are uncoupled from the appropriate behavioral context. Anterior cingulate activity is associated with both attention 29,31 and representation of emotional experience ${ }^{32}$, and the concept of a second-order mapping implies a redundancy in the representation of self, attention and emotion.

By studying perturbations consequent to an uncoupling of integrated autonomic responses in PAF, our findings provide data regarding the neural substrate for dynamic representations of bodily states. The data provide preliminary evidence of an association between peripheral autonomic failure and subtle differences in subjective emotional experience. However, the extent of affective impairments is expected to be limited by the existence of 
established 'as if' representations, and the integrity of sensory feedback regarding other emotion-related bodily responses ${ }^{5}$. There are important non-autonomic pathways of afferent information that contribute to representation of the physical structure of the organism ${ }^{5}$, and these are intact in PAF $^{6}$. Although feedback of autonomic responses is likely to provide a major matrix for on-going representation of bodily states during emotional behavior, it must be emphasized that PAF is acquired late in life, when established patterns of emotional behaviors may be less dependent on direct peripheral feedback. M oreover, the presence of intact hormonal regulatory processes, normal skeletomotor emotional reactions and the integrity of afferent pathways relevant to information about these axes of bodily states is likely to be sufficient to prevent major emotional deficits in subjects with PAF.

In healthy individuals, autonomic responses provide a crucial index of bodily states in support of emotion $3,5,33$ and attention ${ }^{34}$. The proposal that objective feeling of emotion is closely related to mechanisms of bodily homeostasis highlights the importance of further investigation of emotional impairments in patients with pure autonomic failure. The neurophysiological evidence provided in the present study of PAF patients is in accord with data from normal subjects showing that emotional states involve brain regions that contribute to regulation of internal bodily states ${ }^{38}$. The overlap in regions includes pons, somatosensory cortices, insula, caudate nucleus, and anterior and posterior cingulate cortices. Thus, our observations in patients support a neurological model of emotion and consciousness, emphasizing a hierarchy of self-representations rooted in a nested mapping of physical states of the body.

\section{MethodS}

Subjects. Nine subjects ( 5 female; mean age \pm s.d., $66 \pm 6$ years, righthanded) with established diagnoses of $\mathrm{PAF}^{7}$ (greater than five years of symptoms of orthostatic hypotension and dysautonomia, without clinical evidence of non-autonomic pathology or central neurological degeneration, as in cerebellar signs or parkinsonism) were recruited after full clinical assessment, together with eight healthy right-handed age-matched controls ( 4 female; $65 \pm 9$ years; one female control was excluded after discovery of an asymptomatic brainstem malformation). All subjects gave full, informed, written consent to take part in a study approved by the Joint Research Ethics Committee of the National H ospital for Neurology and Neurosurgery and the Institute of Neurology.

Experimental design. Subjects did pseudorandomized repetitions of four experimental tasks $s^{15}$, two of which replicated clinical stressor tests for assessing autonomic function 7 , namely, isometric handgrip exercise (44\% of maximal squeeze strength), and mental arithmetic (serial subtractions of seven, covert during data acquisition). The control exercise task was a low-grade effortless handgrip ( $5 \%$ of maximal squeeze). For the mental arithmetic control, subjects were required to count covertly from a preset value. Task instructions were displayed on a video monitor. To qualitatively assess subjective effort, and ensure accurate performance of the mental arithmetic condition, subjects were systematically debriefed after performance of each task repetition about subjective difficulty and, in the case of the (covert) mathematics conditions, what number they had reached. Heart rate and blood pressure were measured at set times during task performance (just before scan trigger, and at the end of the data acquisition) using Sentron (Bard Biomedical, Lombard, Illinois). Average mean arterial blood pressure (M AP) and heart rate (HR) were calculated for each condition in each subject.

PET scan acquisition and analysis. Scans of the distribution of $\mathrm{H}_{2}{ }^{15} \mathrm{O}$ were obtained using a Siemens/CPS ECAT EXACT HR+ PET Scanner operated in high-sensitivity three-dimensional mode. Subjects received $350 \mathrm{M} \mathrm{bq}$ of $\mathrm{H}_{2}{ }^{15} \mathrm{O}$ over $20 \mathrm{~s}$ through a right antecubital cannula for each of the scans, and activity was measured during a 90-s time window while the subjects did the tasks. The PET images comprised $i, j$ and $k$ voxels $(2 \times 2 \times 3 \mathrm{~mm})$ with a $6.4 \mathrm{~mm}$ transaxial and $5.7 \mathrm{~mm}$ axial resolution (full width at half maximum). The data were analyzed with statistical parametric mapping (SPM 99) ${ }^{35}$, implemented in M atlab (M athworks, Sherborn, M assachusetts). Structural magnetic resonance scans from each subject were cor egistered to the PET data following realignment of the PET time series. All the scans were then transformed ${ }^{36}$ into a standard stereotaxic space ${ }^{37}$. The scans were smoothed using a Gaussian filter set at $12 \mathrm{~mm}$ full width at half maximum. The regional cerebral blood flow measurements were adjusted to a global mean of $50 \mathrm{ml} / \mathrm{dl} / \mathrm{min}$. Between-group differences were examined using random- effects analy$\operatorname{ses}^{17}$. First, to test for task-independent group differences in activity, a design matrix was constructed for analysis of task-by-group interactions, and conjunction analysis $s^{16,17}$ was used to determine commonalities in activity across all tasks. Subsequently, the interaction between diagnosis and task, testing for between-group differences during performance of effortful versus effortless tasks, was examined by constructing a design matrix for the analysis of subject-by-condition interactions. In this analysis, commonalities were identified between exercise and mathematics contrasts to determine between-group differences that emerged during effort per se. As SPM corrects for the entire volume, and to avoid Type 2 errors for a priori regions of interest, small volume correction was done and significance was corrected for volume of region of interest (that is, cingulate and cingulate + medial parietal, thalamus and superior colliculi) in the examination of diagnosis-by-effort interaction. Thus, we tested specific brain areas predicted a priori using a highly conservative random-effects analysis, thereby ensuring that Type 1 errors were unlikely to have occurred.

Evaluation of subjective emotional experience. Our original screening excluded any subject with overt present or past psychiatric disorder. We used standard clinical screening tools to rate physical disability (Activities of Daily Living Q uestionnaire, $A D L^{18}$ ) and anxiety symptoms (H amilton Anxiety Scale, HAM A ${ }^{19}$ ) in PAF patients and controls who took part in the PET imaging study. In addition, because physical symptoms are a likely confounding factor in the analysis of mood symptoms, we also compared PAF patients with nine patients with idiopathic Parkinson's disease (IPD) who were matched with the PAF patients on degree of disability, indexed by the ADL ${ }^{18}$. Wealso expanded our analysis to include larger groups of PAF patients $(n=28)$, controls $(n=15)$ and IPD patients $(n=13)$. In this expanded comparison between PAF and IPD patients, disability ( $A D L$ ) score was treated as a confounding covariate. M ore selective assessments of subjective emotional awareness were carried out at the clinical interview. Additionally, subjects were asked to rate statements related to specific affective symptoms expected to arise from absence of autonomic emotional responses. Subjects rated (using a 5point Likert scale) four accuracy statements: "I can no longer feel sadness," "I have lost my ability to feel emotional," "I feel distant from everyone," and "I feel I can no longer control my emotions."

\section{ACKNOWLeDGements}

This work was supported by a ProgrammeGrant to R.J.D. from the Wellcome Trust. H.D.C. is supported by a Clinical Research Fellowship from the Brain Research Trust.

\section{ReCeived 30 Ctober; Accepted 27 November 2000}

1. James, W. Physical basis of emotion. Psychol. Rev. 101, 205-210 (1994)

2. Damasio, A. R. Descartes' Error: Emotion, Reason and the Human Brain (Grosset/Putnam, New York, 1994).

3. Bechara, A., Damasio, H., Tranel, D. \& Damasio, A. R. Deciding advantageously before knowing the advantageous strategy. Science 275, 1293-1295 (1997)

4. Cahill, L. The neurobiology of emotionally influenced memory. Implications for understanding traumatic memory. Ann. NY Acad. Sci. 821, 238-246 (1997).

5. Damasio, A. R. The Feeling of What Happens: Body and Emotion in the M aking of Consciousness (H arcourt Brace, New York, 1999). 
6. Mathias, C. J. in Neurology in Clinical Practice (eds. Bradley, W. G., Daroff, R. B., Fenichel, G. M . \& M arsden, C. D.) 2131-2165 (Butterworth-Heinemann, Woburn, Massachusetts, 2000).

7. Mathias, C. J. \& Bannister, R. in Autonomic Failure: A Textbook of Clinical Disorders of the Autonomic Nervous System 4th edn. (eds. Mathias C. J. \& Bannister, R.) 169-195 (Oxford Univ. Press, Oxford, 1999).

8. Mathias, C. J., M allipeddi, R. \& Bleasdale-Barr, K. Symptoms associated with orthostatic hypotension in pure autonomic failure and multiple system atrophy. J. Neurol. 246, 893-898 (1999).

9. Magnifico, F. Misra, V. P. M urray, N. M. \& Mathias, C. I. The sympathetic skin response in peripheral autonomic failure- evaluation in pure failure pure cholinergic dysautonomia and dopamine-beta-hydroxylase deficiency. Clin. Auton. Res. 8, 133-138 (1998).

10. Clark, C. V. \& Ewing, D. J. Ocular autonomic function in progressive autonomic failure. D oc. O phthalmol. 70, 309-321 (1988).

11. Matthews, M. in Autonomic Failure: A Textbook of Clinical Disorders of the Autonomic Nervous System 4th edn. (eds. Mathias C. J. \& Bannister, R.) 169-195 (Oxford Univ. Press, Oxford, 1999).

12. Kimber, J. R., Watson, L. \& Mathias, C. J. Distinction of idiopathic Parkinson's disease from multiple-system atrophy by stimulation of growthhormone release with clonidine. Lancet 349, 1877-1881 (1997).

13. Colebatch, J. G., Deiber, M. P., Passingham, R. E., Friston, K. J. \& Frackowiak, R. S. Regional cerebral blood flow during voluntary arm and hand movements in human subjects. J. Neurophysiol. 65, 1392-1401 (1991).

14. Paulesu, E., Frith, C. D. \& Frackowiak, R. S. The neural correlates of the verbal component of working memory. Nature 362, 342-345 (1993).

15. Critchley, H. D., Corfield, D. R., Chandler, M. P., M athias, C. J. \& Dolan, R. J. Cerebral correlates of autonomic cardiovascular arousal: A functional neuroimaging investigation. J. Physiol. (Lond.) 523, 259-270 (2000).

16. Price, C. J. \& Friston, K. J. Cognitive conjunction: a new approach to brain activation experiments. Neuroimage 5, 261-270 (1997).

17. Friston, K. J., Holmes, A. P., Price, C. J., Buchel, C. \& Worsley, K. J. Multisubject fMRI studies and conjunction analyses. Neuroimage 10, 385-396 (1999).

18. Katz, S., Ford, A. B., M oskowitz, R. W., Jackson, B. A. \& Jaffe, M. W. Studies of illness in the aged. The Index of ADL: a standardized measure of biological and psychosocial function. JAM A 185, 914-919 (1963).

19. Hamilton, M. Diagnosis and rating of anxiety. Br. J. Psychiatry, Special Publication No. 3, 76-79 (1969).

20. Xi, M. C., M orales, F. R. \& Chase, M . H. Evidence that wakefulness and REM sleep are controlled by a GABAergic pontine mechanism. J. Neurophysiol. 82, 2015-2019 (1999).

21. Spyer, K. M. in Autonomic Failure: A Textbook of Clinical Disorders of the Autonomic Nervous System 4th edn. (eds. M athias C. J. \& Bannister, R.) 45-55
(Oxford Univ. Press, Oxford 1999).

22. Chamberlin, N. L.\& Saper, C. B. Topographic organization of cardiovascular responses to electrical and glutamate microstimulation of the parabrachial nucleus in the rat. J. Comp. Neurol. 326, 245-262 (1992).

23. Saleh, T. M., Bauce, L. G. \& Pittman, Q. J. Glutamate release in parabrachial nucleus and baroreflex alterations after vagal afferent activation. Am. J. Physiol. 272, R1631-R1640 (1997)

24. van der Plas, J., Maes, F. W. \& Bohus, B. Electrophysiological analysis of midbrain periaqueductal gray influence on cardiovascular neurons in the ventrolateral medulla oblongata. Brain Res. Bull. 38, 447-456 (1995).

25. Cechetto, D. F. \& Saper, C. B. Evidence for a viscerotopic sensory representation in the cortex and thalamus in the rat. J. Comp. Neurol. 262, 27-45 (1987).

26. Craig, A. D., Chen, K., Bandy, D. \& Reiman, E. M. Thermosensory activiation of insula cortex. Nat. Neurosci. 3, 184-190 (2000).

27. Adolphs, R. et al. A role for somatosensory cortices in the visual recognition of emotion as revealed by three dimensional lesion mapping. J. N eurosci. 20, 2683-2690 (2000)

28. Critchley, H. D., Elliott, R., Mathias, C. J. \& Dolan, R. J. Neural activity relating to the generation and representation of galvanic skin conductance response: a functional magnetic imaging study. J. Neurosci. 20, 3033-3040 (2000).

29. Devinsky, O., Morrell, M . J. \& Vogt, B. A. Contributions of anterior cingulate cortex to behaviour. Brain 118, 279-306 (1995).

30. Vogt, B. A., Finch, D. M . \& Olson, C. R. Functional heterogeneity in cingulate cortex: the anterior executive and posterior evaluative regions. Cereb. Cortex 6, 435-443 (1992)

31. Cohen, R. A., Kaplan, R. F., Moser, D. J., Jenkins, M. A. \& Wilkinson, H. Impairments of attention after cingulotomy. Neurology 53, 819-824 (1999).

32. Lane, R. D., Fink, G. R, Chau, P. M. \& Dolan, R. J. Neural activation during selective attention to subjective emotional responses. Neuroreport $\mathbf{8}$, 3969-3972 (1997).

33. Collet, C., Vernet-Maury, E., Delhomme, G. \& Dittmar, A. Autonomic nervous system response patterns specificity to basic emotions. J. Auton. Nerv. Syst. 62, 45-57 (1997)

34. Bouscein, W. Electrodermal Activity (Plenum, New York, 1992).

35. Friston, K. et al. Statistical parametric maps in functional imaging: a general linear approach. Hum. Brain Mapp. 2, 189-210 (1995)

36. Friston, K. et al. Spatial registration and normalization of images. Hum. Brain M app. 2, 165-189 (1995).

37. Talairach, J. \& Tournoux, P. Co-planar Stereotaxic Atlas of the Human Brain (Theime, Stuttgart, Germany, 1988)

38. Damasio, A. R. et al. Subcortical and cortical brain activity during the feeling of self-generated emotions. Nat. N eurosci. 10, 1049-1056 (2000). 